\title{
482.
}

\section{NOTE ON A PAIR OF DIFFERENTIAL EQUATIONS IN THE LUNAR THEORY.}

[From the Monthly Notices of the Royal Astronomical Society, vol. xxxir. (1871-72), pp. 31-32.]

THE equations

$$
\begin{array}{ll}
\frac{d}{d t} \frac{d \rho}{d t}-\rho\left(\frac{d v}{d t}\right)^{2}+\frac{1}{\rho^{2}} & =k m^{2} \rho\left\{\frac{1}{2}+\frac{3}{2} \cos (2 v-2 m t)\right\}, \\
\frac{d}{d t} \rho^{2} \frac{d v}{d t} & =j m^{2} \rho^{2}\left\{-\frac{3}{2} \sin (2 v-2 m t)\right\},
\end{array}
$$

taking therein $j=k=1$ in effect present themselves in the Lunar Theory, and particular integrals in series have been obtained, the development being carried to a great extent; but I give the results only as far as $m^{4}$, viz., writing

we have

$$
t-m t=D \text {, }
$$

$$
\begin{aligned}
v=t & +\left(\frac{11}{8} m^{2}+\frac{59}{12} m^{3}\right. \\
& \left.+\frac{893}{72} m^{4}\right) \sin 2 D \\
& +\frac{201}{256} m^{4} \sin 4 D \\
\frac{1}{\rho}=1+\frac{1}{6} m^{2} & -\frac{179}{28} m^{4} \\
+\left(m^{2}+\frac{19}{6} m^{3}\right. & \left.+\frac{131}{18} m^{4}\right) \cos 2 D \\
& +\frac{7}{8} m^{4} \cos 4 D
\end{aligned}
$$

In the Lunar Theory $j$ and $k$ are properly each $=\frac{1}{1+\frac{E}{m^{\prime}}}(E$ the mass of the

Earth, $m^{\prime}$ that of the Sun), but they are taken to be $=1$; the numerical difference is inappreciable; but there would be a considerable theoretical advantage in retaining 
in the equations the coefficients $j, k$ [regarded as each of them $=k$ ]: in fact, the developments could then be arranged according to the powers of $k$, that is according to the powers of the disturbing force; whereas, when $k$ is taken $=1$, we have only a development in powers of $m$, and since $m$ also presents itself through the coefficient $2-2 m$ of $t$ in $2 v-2 m t$, terms which are really of different orders in regard to the disturbing force, are united together into a single term: so that, instead of a term of the form $\left(A k+B k^{2}+\& c\right.$. $) m^{p}$, where $A, B$, are numerical, we have the term $(A+B+\ldots) m^{p}$, where of course $A+B .$. is given as a single numerical coefficient. There is no equal advantage in retaining the two coefficients $k, j$, as this only serves to show how a term arises from the central and tangential forces respectively; thus retaining these coefficients, the integrals as far as $m^{2}$ are

$$
\begin{aligned}
& v=\quad t \quad+\left(\frac{1}{2} k+\frac{7}{8} j\right) m^{2} \sin 2 D, \\
& \frac{1}{\rho}=1+\frac{1}{6} m^{2} k+\left(\frac{1}{2} k+\frac{1}{2} j\right) m^{2} \cos 2 D,
\end{aligned}
$$

agreeing with the former result when $k=j=1$; but there is, nevertheless, some interest in retaining the two coefficients. I hope to develope the results somewhat further, and to communicate them to the Society. 